\title{
Insight into improving antidepressant adherence and symptoms by pharmacist intervention: A review
}

\author{
Mohammed Hassen ${ }^{1}$, Yibing $\mathrm{Li}^{2 \star}$, Xinyue Yang ${ }^{2}$, Umeorah Ernest ${ }^{3}$ \\ ${ }^{1}$ Department of Social and Management Pharmacy, International Pharmaceutical Business School, China Pharmaceutical \\ University, 639 Longmian Avenue, Jiangning District, Nanjing 211198, ${ }^{2}$ Department of Social and Management Pharmacy, \\ ${ }^{3}$ Department of Biomedical Engineering, School of Engineering, China Pharmaceutical University, 24 Togjia Lane, Gulou \\ District, Nanjing 210009, China
}

*For correspondence: Email: Zgykdxlyb@sina.com, Tel: +8613347737072

\begin{abstract}
Purpose: To assess the effectiveness of antidepressant medication adherence-improving intervention by a pharmacist and its impact on clinical symptoms of depression among outdoor depressive patients. Methods: Various databases such as PubMed, Embase, and Scopus were used sources for the literature published during the last 20 years. Pharmacist intervention studies involving adult depressed patients ( $\geq 17$ years old) and treated with antidepressants were included. Twelve studies met the inclusion criteria.

Results: These studies depicted various levels of interventions in which pharmacist counseled and educated the patients to support medication adherence. In only one of the studies, pharmacist intervention exercised significant effect on the depression features of patients.

Conclusion: The findings suggest that the implication of antidepressant medication adherenceimproving intervention by pharmacist leads to the improved adherence of adult depressive patients to antidepressants. However, pharmacist intervention did not show any significant influence on depression symptomology, necessitating further studies on the topic.
\end{abstract}

Keywords: Pharmacist care, Depression, Antidepressants, Intervention, Medication adherence

\begin{abstract}
This is an Open Access article that uses a funding model which does not charge readers or their institutions for access and distributed under the terms of the Creative Commons Attribution License (http://creativecommons.org/licenses/by/4.0) and the Budapest Open Access Initiative (http://www.budapestopenaccessinitiative.org/read), which permit unrestricted use, distribution, and
\end{abstract} reproduction in any medium, provided the original work is properly credited.

Tropical Journal of Pharmaceutical Research is indexed by Science Citation Index (SciSearch), Scopus, International Pharmaceutical Abstract, Chemical Abstracts, Embase, Index Copernicus, EBSCO, African Index Medicus, JournalSeek, Journal Citation Reports/Science Edition, Directory of Open Access Journals (DOAJ), African Journal Online, Bioline International, Open-J-Gate and Pharmacy Abstracts

\section{INTRODUCTION}

Depression is one of the most prevalent mental health disorder worldwide [1-3] and will likely become the second largest reason for health illness globally by the year 2020 [4]. Only in the USA, several billions of cost is associated with depression disorders [5]. Antidepressant agents are primarily used to manage depression [6]. Although depression can be effectively treated with antidepressants, patient nonadherence to medication is a substantial barrier to treatment success in clinical practice [7,8]. World Health Organization (WHO) has included depression to top 10 chronic disorders, which require medication adherence [9]. Some studies have revealed a significant positive effect of hospital admission on medication nonadherence [10].

Medication adherence refers to a behavioral feature, which is altered with the passage of 
time. This change could be a function of the changing attitudes of patients towards prescribed medication [11]. Medication adherence is a component of a decision-making process, in which patients weigh their hesitations against the apparent benefits and make decisions about their medication [12]. During the last twenty years, a significant increase in the patient's decisionmaking authority has been observed $[13,14]$.

Patients with depressive disorder have shown curiosity in improving their knowledge about treatment plans and taking part in treatment decisions $[15,16]$. Contrary to this condition, psychiatrist-patient interaction is not enough for making suitable treatment decisions [17]. Studies have demonstrated that adherence, satisfaction, and clinical outcomes can be improved by the active participation of patients in decision-making for treatment of depression.

The pharmacist is one of the most accessible health-care professionals, mainly for outpatients suffering from various clinical abnormalities, such as asthma, diabetes, and depression [18]. Thus pharmacist has significant involvement in outpatient intervention, which subsequently helps in medication adherence and reduces hospital admissions [19,20]. Pharmacist interventions involve patient counseling and education, mainly on medication adherence, clinical symptomology, treatment outcomes, and prescription modification. The educational materials comprise of audios, videos, and brochures. The interventions are generally performed through phone or in-person.

Various qualitative and quantitative studies have reported mixed findings of the effectiveness of pharmacist intervention toward the improved adherence to antidepressant medication and beneficial clinical outcome. For instance, patients who received pharmacist intervention showed better adherence to medication than the control group subjects [21]. Another publication reported the useful outcomes of pharmacist intervention for better adherence to medication used for depression [22].

The objective of writing this review is to accumulate the old and recent studies (randomized controlled trials) on the effectiveness of pharmacist counseling on adherence to medication used for depression and clinical symptoms of depression among depressed outpatients. It was assumed that medication adherence and clinical symptoms of depression might be influenced by study protocols.

\section{METHODS}

To assess the effect of pharmacist intervention on the improvement of adherence to antidepressant medication and clinical symptoms of depression among outdoor depressive patients, a broad electronic literature search (published during last 20 years) was made using various databases including PubMed, Embase, and Scopus. For searching literature, various text words such as "pharmacist intervention", "adherence to medication used for depression", "quality of life" and "clinical symptoms of depression among outdoor depressive patients" were used. The inclusion criteria for this assessment were as follows: studies involving pharmacist intervention with an aim of getting improved adherence to medication and clinical symptoms of depression, controlled trials (both randomized and non-randomized) in the presence of a control group, and adult patients with confirmed condition of depression $(\geq 17$ years old), outdoor healthcare settings. Research publications published in only English language were included. The retrieved literature was validated for duplication, which if detected were excluded. As a result, 12 publications were identified and included for this review.

\section{RESULTS AND DISCUSSION}

Medication adherence is an indicator of two pharmaceutical terms, i.e. persistence and compliance. The former means medication use during the course of treatment [23], while the latter term represents medication use with the instructed directions [24]. However, these two terms are used interchangeably.

\section{Factors affecting adherence}

Factors related to the patient, clinical setting, social behavior, and medication affect are involved in patient adherence. A variety of patient factors including patient beliefs, fear of addiction, and worries about the undesired effects affect adherence. Poor follow-up and patient illiteracy are the clinical factors causing non-adherence. Moreover, social factors such as poverty and poor social support also act as barriers to adherence. In addition, undesired effects and medication cost are the drug factors influencing adherence [25-30]. Non-adherence results in disease recurrence augmented severity of depression and re-hospitalizations.

The factors affecting medication use include the probable undesired effects and the desired efficacy [31]. During the dosage regimen design of antidepressants, the prescriber should take 
care of the probable side effects, since the emergence of undesired effects influences the patient's confidence in the prescriber and medication [32]. Adherence was found to be independent of sociodemographics such as age and sex; however, poor psychological status, poverty, and excessive medicine prescription had a significant association with adherence [33]. Other authors correlated antidepressant compliance with race, age, and sex [34,35]. Another study showed better adherence in females [36]. However, low-income females exhibited worse compliance [37,38]. Thus, the fear of undesired effects or disease relapse as well as, the anxiety of becoming addicted and the increased expenses leads to medication nonadherence.

\section{Measurement of adherence}

According to literature, $30-60 \%$ of depressed patients do not exhibit adherence to antidepressant medication [39]. This prevalence of poor adherence is not lowered after introducing newer antidepressants having reduced side effects. The problem of noncompliance is not a serious issue for a large number of physicians. Regardless of the improved therapeutics, reduced undesired effects, treatment algorithms, and doing clinical practices according to the latest guidelines, different antidepressants show different efficacy [40]. This difference may be because of poor information.

Patient's awareness about medication side effects enhances adherence, thus patients can improve adherence by remembering the physician's advice [41]. Moreover, there could be a disagreement between what the physician instruct and what the patient remembers.

Averagely, patients with poor compliance discontinue medication use after only 43 days, notwithstanding positive response of medication. However, the emergence of side effects after 15 days of initiating medication use causes poor compliance, while non-compliance is seen after 20 days if a positive outcome is not experienced [42]. Thus the compliance can be assumed to be associated with the therapeutic outcomes.

According to a study on the beliefs of psychiatrists, the chronically depressive patients exhibit a low prevalence of non-adherence, i.e. $16 \%$ in this study [34]. It is the usual perception that the depressed people who voluntarily visit the physician for consultation follow the prescription. Approximately $33 \%$ of the depressed patients admit that they "very often" make mistake in taking medication. Factors involved in effective medication include the promising efficacy of the drug and a low number of pills per day. Adherence is improved by family and physician's support, emotional well-being, and the proposed positive outcome of medication. However, the prolonged duration of therapy adversely affects the adherence.

\section{Barriers to adherence}

According to a study, a majority (50\%) of the patients do not fully understand the physician's instructions on medication before leaving his clinic [43]. Moreover, low education level and healthcare accessibility badly influence treatment use [44]. It can be submitted that low social and cultural levels are the social and demographic factors that cause forgetfulness in medication causing non-adherence.

Antidepressant medication adherence with chronic comorbidities is complex [31]. The concomitant use of various drugs may result in unexpected side effects and low efficacy due to drug interaction causing non-compliance as well [31]. Additionally, improvement in somatic disorder symptoms is another aspect that results in the management of various psychological disorders such as depression. It is an established fact that depression is related to non-adherence and increased morbidity and mortality [45], while antidepressant adherence reduces the threat of mortality [46]. Depression may cause an unlikely deficit of faith triggering therapeutic noncompliance for somatic disorders [47]. Prior to adopting a more intensive medication plan in depression, the assessment of adherence, compliance, and persistence is advised, since $50 \%$ of all the depressed patients for whom a second line medication is required are nonadherent.

\section{Adherence improvement by healthcare providers}

It is expected that improved response to treatment is associated with the prescription of better treatment, however, this must be remembered at the time of prescribing medication. Patients deserve to be informed of the medication efficacy, side effects, and interaction as well as of the lag time for therapeutic benefits. The poorer the antidepressant medication compliance, the worse is the outcome with more recurrences and increased cost [36]. Poor compliance may be caused by confusion or scarcity of medical information: Generally, patients do not continue treatment on feeling better, addiction and/or fear 
of the undesired effects. In view of all of the above, a physician should address all patient queries regarding drug treatment, rather patient should insist on seeking medical information.

A physician chooses medication on the basis of the severity of the disease as well as according to his/her perception. For instance, anxiety disorders are effectively treated with antidepressants. If the patient knows this fact, antidepressant use could be improved. Positive patient behavior towards antidepressants in the first month of their use could reduce early side effects [23] leading to adherence improvement, particularly persistence, though the prolonged consumption is not indicated.

Considering the benefits of adherence and their practical outcomes, adequate measurement of medication use is necessary, so that the collected information may be utilized to develop policies that solve this issue in the general public. It is difficult to measure the intervention effect on antidepressant medication in depressed patients with chronic comorbidities, and thus the effect is independent of the level of intervention complexity $[48,49]$. The studies have reported no definite clues of which interventions may be useful.

However, due to the above-mentioned difficulties, the efforts to tackle this issue should not be abandoned, since non-adherence may lead to treatment failure of depression resulting in clinical, social and economic problems [50]. Thus, to improve adherence, healthcare providers such as pharmacist should implement suitable strategies, for instance, patient education, telephonic or in-person follow-up and providing medication.

\section{Pharmacist intervention and antidepressant medication adherence}

The findings of previous studies reflect an effective influence of pharmacist intervention on adherence to antidepressants medication in adult outpatients with depression. Table 1-2 describes the adherence outcomes observed for both $<6$ months and 6 months. The outcomes noted in 6months study showed more variability in outcomes than that of observed in $<6$ months study. Approximately 2.5 times more adherence to antidepressants medication was observed in the intervened group of depressed patients than the patients' group who were given usual pharmacist care only [20,21,51-56]. The comparison groups did not show any significant difference in adherence in spite of differences in methodological characteristics (Table 1). However, adherence is also affected by the outpatient clinical setting and location. As far as location is concerned, medication adherence was lower in the American outpatients than the Middle-Eastern outpatients, arguing potentially more effective intervention in the Middle-East studies [20,53]. In the case of settings, profoundly higher adherence was noted in outpatient clinics than the primary care practice settings. Thus, it is critical to consider the influence of setting difference on pharmacist intervention, arguing the need for further studies.

Table 1: Study features for the assessment of the impact of pharmacist intervention on antidepressant therapy

\begin{tabular}{|c|c|c|c|c|c|c|c|}
\hline \multirow[t]{2}{*}{ Country } & \multirow{2}{*}{$\begin{array}{l}\text { Healthcare } \\
\text { setting }\end{array}$} & \multicolumn{2}{|c|}{ Study group } & \multirow{2}{*}{$\begin{array}{c}\text { Study } \\
\text { design }\end{array}$} & \multirow{2}{*}{$\begin{array}{c}\text { Follow-up } \\
\text { time } \\
\text { (months) }\end{array}$} & \multirow{2}{*}{$\begin{array}{l}\text { Type of } \\
\text { analysis }\end{array}$} & \multirow[t]{2}{*}{ Ref } \\
\hline & & $\begin{array}{l}\text { Usual care } \\
\text { group (n) }\end{array}$ & $\begin{array}{c}\text { Intervention } \\
\text { group }(n)\end{array}$ & & & & \\
\hline KSA & PCP & 110 & 110 & RCT & 6 & ITT & 51 \\
\hline Kuwait & OpC & 87 & 87 & RCT & 2,5 & ITT & 53 \\
\hline Kuwait & OpC & 87 & 87 & RCT & 2,5 & ITT & 53 \\
\hline USA & $\mathrm{CP}$ & 33 & 41 & RCT & 6 & ITT & 57 \\
\hline USA & PCP & 268 & 533 & RCT & 6 & PP & 58 \\
\hline USA & PCP & 129 & 91 & $\begin{array}{l}\text { Non- } \\
\text { RCT }\end{array}$ & 6 & ITT & 52 \\
\hline USA & $\mathrm{CP}$ & 50 & 75 & RCT & 6 & ITT & 59 \\
\hline USA & $\mathrm{CP}$ & 32 & 31 & RCT & 3 & PP & 60 \\
\hline Australia & $\mathrm{CP}$ & 68 & 51 & PGD & 2 & PP & 61 \\
\hline Spain & $\mathrm{CP}$ & 87 & 92 & RCT & 6 & ITT & 54 \\
\hline Netherlands & $\mathrm{CP}$ & 71 & 64 & RCT & 6 & ITT & 62 \\
\hline Israel & $\mathrm{CP}$ & 12,746 & 173 & $\begin{array}{l}\text { Non- } \\
\text { RCT }\end{array}$ & & PP & 48 \\
\hline Brazil & $\begin{array}{c}\text { OpC and } \\
\text { patient homes }\end{array}$ & 27 & 31 & RCT & 3 & PP & 63 \\
\hline
\end{tabular}

Key: USA = United States of America, KSA = Kingdom of Saudi Arabia; PCP $=$ Primary care practice, OpC = Outpatient clinic, $\mathrm{CP}=$ Community pharmacy, $\mathrm{RCT}=$ Randomized controlled trials, Non-RCT = Non-randomized controlled trials, PGD = Parallel groups design; PP = Per-protocol, ITT = Intention-to-treat 
Table 2: Study protocols for the determination of the influence of pharmacist counseling on antidepressant therapy and qualitative summary of outcomes of depression symptoms

\begin{tabular}{|c|c|c|c|c|}
\hline $\begin{array}{l}\text { Study } \\
\text { ref }\end{array}$ & $\begin{array}{l}\text { Intervention } \\
\text { elements }\end{array}$ & $\begin{array}{l}\text { Adherence } \\
\text { determination }\end{array}$ & $\begin{array}{l}\text { Symptomology } \\
\text { determination }\end{array}$ & $\begin{array}{l}\text { Qualitative summary of outcomes of depression } \\
\text { symptoms }\end{array}$ \\
\hline 51 & EM & SR on MARS & MADRS & $\begin{array}{l}\text { No significant difference in depression symptoms } \\
\text { between Usual care and intervention group }\end{array}$ \\
\hline 53 & $\mathrm{~B}$ & $\begin{array}{l}\text { SR, Counting of } \\
\text { pills }\end{array}$ & - & Not measured \\
\hline 53 & $A, B$ & $\begin{array}{l}\text { SR, Counting of } \\
\text { pills }\end{array}$ & - & Not measured \\
\hline 57 & $\begin{array}{l}\text { AC, Tt, Tp, MDR, } \\
\text { MCS, MTO, PMP, } \\
\text { SCM, PoT }\end{array}$ & SR & SCL-20 & $\begin{array}{l}\text { Depression symptomology was improved in both } \\
\text { groups }\end{array}$ \\
\hline 58 & $\begin{array}{l}\text { AC, Tt, Tp, MDR, } \\
\text { MCS, PMP, PoT, } \\
\text { AIA }\end{array}$ & $\begin{array}{l}\text { SR on } \\
\text { questionnaire }\end{array}$ & mBDI & $\begin{array}{l}\text { Lower depression symptoms in the intervention } \\
\text { group }\end{array}$ \\
\hline 52 & $\begin{array}{l}\text { AC, Tt, MM, Tp, } \\
\text { MDR, MCS, MTO, } \\
\text { PMP, SCM,B }\end{array}$ & PR & $\begin{array}{l}\text { Clinician-related } \\
\text { IDS }\end{array}$ & $\begin{array}{l}\text { Lower depression symptoms in the intervention } \\
\text { group }\end{array}$ \\
\hline 59 & $\begin{array}{l}\text { AC, Tt, MM, Tp, } \\
\text { MDR, MCS, MTO, } \\
\text { PMP, SCM }\end{array}$ & PR & Brief IDS & $\begin{array}{l}\text { No significant difference in depression symptoms } \\
\text { between Usual care and intervention group, but } \\
\text { depression symptomology was improved in both } \\
\text { groups. }\end{array}$ \\
\hline 60 & $\begin{array}{l}\text { AC, Tt, A, Tp, } \\
\text { MDR, MCS, MTO, } \\
\text { PMP, AIA }\end{array}$ & PR & BDI-II & $\begin{array}{l}\text { No significant difference in depression symptoms } \\
\text { between Usual care and intervention group }\end{array}$ \\
\hline 61 & $\begin{array}{l}\text { TV, Tt, A, MDR, } \\
\text { MCS, AIA,B }\end{array}$ & SR & K10 & $\begin{array}{l}\text { Depression symptomology was improved in both } \\
\text { groups, but no significant difference in depression } \\
\text { symptoms between Usual care and intervention } \\
\text { group. }\end{array}$ \\
\hline 54 & $\begin{array}{l}\text { AC, Tt, MDR, } \\
\text { MCS, MTO, AIA }\end{array}$ & PR & PHQ-9 & $\begin{array}{l}\text { No significant difference in depression symptoms } \\
\text { between Usual care and intervention group }\end{array}$ \\
\hline 62 & $\mathrm{~T}, \mathrm{AC}, \mathrm{TV}$ & eDEM & SCL-13 & $\begin{array}{l}\text { No effect on depression symptoms in intervention } \\
\text { group }\end{array}$ \\
\hline 48 & $\begin{array}{l}\text { AC, Tt, A, MDR, } \\
\text { MCS }\end{array}$ & PR & - & Not measured \\
\hline 63 & $\mathrm{Tt}, \mathrm{B}, \mathrm{DM}$ & - & BDI & $\begin{array}{l}\text { A significant difference in depression symptoms } \\
\text { between Usual care and intervention group }\end{array}$ \\
\hline
\end{tabular}

Key: MARS = Morisky Medication Adherence Scale, MADRS = Montgomery-Äsberg Depression Rating Scale, mBDI = Modified Beck Depression Inventory; Dash (-) means "not measured"; Dash (-) means "not measured"; $\mathrm{SR}=$ Self-report, eDEM = Electronic pill container, $\mathrm{PR}=$ Pharmacy record; SCL-13 = Hopkins Symptom Checklist depression subscale, SCL-20 = Hopkins Symptom Checklist, K10 = Kessler Psychological Distress Scale, IDS = Inventory for depressive symptoms, BDI = Beck depression inventory, $\mathrm{PHQ}=\mathrm{Psychological}$ health questionnaire; $\mathrm{T}$ = Training, $\mathrm{AC}=$ Adherence campaign, $\mathrm{TV}=$ Training videos, $\mathrm{MM}=$ Management of medication, $\mathrm{Tt}=$ Tutoring, $\mathrm{A}=$ Advising, $\mathrm{Tp}=$ Telephonically, $\mathrm{MDR}=$ Monitoring of drug reactions, $\mathrm{MCS}=$ Monitoring of clinical symptoms, MTO = Monitoring of treatment objectives, PMP = Protocol based modification in prescription, $\mathrm{SCM}=$ Suggested modification in medication, $\mathrm{DM}=$ Dader method, PoT = Provision of other treatments, AIA = Additional instruction and assistance, $\mathrm{B}=$ Brochure, $\mathrm{EM}=$ Education meetings

The differential effects of setting and location cannot be determined since both are the confounders. Similarly, the differential effects of pharmacist intervention on medication adherence cannot be easily correlated with other characteristics including study design and quality, intervention components, and adherence determinants, since the differences in these characteristics are small [21]. The documented studies were found to have a medium and high level of bias. To recognize the relationship between the risk of bias and effect size in the area, further studies are required. Additionally, all the educational materials including audios, videos, and brochures were found to have a promising effect on medication adherence in all studies [20-22]. The identified differences can be further explored in future studies. Moreover, the difference in the treatment outcomes could be due to several factors including setting difference, protocol differences in different population groups, factors associated with patients, intra-population variability, and the level of a system.

\section{Pharmacist intervention and clinical symptoms of depression}

In most of the reported studies, clinical outcome was demonstrated by documenting the level of 
depression symptoms. Regardless of different types of validated lists of symptom across the intervention studies to determine depression symptomology, only one of the 12 studies [63] documented profound influences of the intervention on depression symptomology. The findings from previous studies did not show any evidence whether clinical symptoms of depression were profoundly affected by pharmacist intervention. Both the usual care groups and intervention groups reflected the similar extent of improvement in clinical symptoms of depression from baseline in most studies [51,56,57,59,61], except one which showed a substantial difference between the two groups after a follow-up of three months [62]. It proposes that substantial efficacy of antidepressant medication was achieved, and nine of 12 studies depicted profoundly improved medication adherence, but clinical symptoms of depression were not significantly improved as a result of about 6-month follow-up by pharmacist [22-54,64].

The remaining two studies showed a positive clinical effect in the intervened patients [52,58]. One of these two studies depicted better therapeutic outcome in the counseled patients, but there was a non-significant difference between the follow-up scores of the control and intervention group [58]. The other study did not contain a control group for comparison with intervention one (Table 2) [52].

It is also evident from the literature that there was a more useful outcome of an intervention on clinical symptoms in studies with the longer follow-up period [19]. Thus studies with longer follow-up periods should be carried out in future to achieve long-term clinical outcomes in depressive patients being treated with antidepressants.

The collaborative care plans involving pharmacist and other healthcare professionals' intervention have also shown a positive effect on depression symptomology. Remarkably, the clinical symptoms of the depressed patients were significantly improved when the studies were carried out under the consistent direction of the case manager trained in mental health. It argues that pharmacist intervention alone may be sufficient for improvement in medication adherence, but clinical improvement may also be achieved by coupling a pharmacist with a mental health case manager. However, such an outcome may be achieved by developing an interprofessional interaction. Otherwise, medication adherence and clinical symptomology may be more effectively improved if pharmacists adopt a standardized intervention strategy, for instance, health coaching. Future studies can be focused on these areas. At all, the studies included in this review unsuccessfully exhibited profound clinical improvements in clinical symptoms of depression associated with the pharmacist intervention.

\section{Limitations of the study}

The diversity of methods adopted for the measurement of antidepressant adherence in the identified publications is an essential limitation. The variety of methodologies influence the quality of these studies and generalization of the extracted results. In addition, most studies did not hide the identity of the control and the intervened group subjects from pharmacists. They may have obviously been persuaded to render more attention to the control group subjects who were pursuing additional assistance. However, this performance bias has been addressed in some studies by utilizing the services of blind raters.

\section{CONCLUSION}

The promising role of pharmacists in antidepressant medication adherence has a valuable impact on the healthcare system worldwide. Owing to direct interaction with outdoor patients suffering from various ailments including depression, pharmacists' role is increasingly being incorporated into healthcare reforms. The present review article urges further studies encompassing long follow-up period for a better description of pharmacists' role in achieving better health of depressive patients and eventually suppressing the financial liability related to the disease nature. Thus, the use of antidepressant medication adherence-improving intervention by a pharmacist results in better adherence by adult depressive patients to antidepressants. Nonetheless, pharmacist intervention did not reveal any beneficial effect on depression symptomology.

\section{DECLARATIONS}

\section{Acknowledgement}

The authors wish to acknowledge Yibing Li for his guidance and support throughout this paper.

\section{Conflict of interest}

The authors declare that there is no conflict of interest with regard to this study. 


\section{Contribution of authors}

The authors declare that this work was done by the authors named in this article and all liabilities pertaining to claims relating to the content of this article will be borne by them. All the authors were involved in conceiving and designing the study as well as drafting the manuscript and revising it critically for crucial contents. All the authors worked for acquisition and interpretation of data and approved the final manuscript.

\section{REFERENCES}

1. Demyttenaere $K$, Bruffaerts $R$, Posada-Villa $J$. Prevalence, severity, and unmet need for treatment of mental disorders in the World Health Organization World Mental Health Surveys. J Am Med Assoc 2004; 291: 2581-2590.

2. Mathers $C D$, Loncar $D$. Projections of global mortality and burden of disease from 2002 to 2030. PLoS Med 2006; 3: e442-e447.

3. Sobocki P, Jonsson B, Angst J, Rehnberg C. Cost of depression in Europe. J Ment Health Policy Econ 2006; 9: 87-98.

4. Murray CJ, Lopez AD. Alternative projections of mortality and disability by cause 1990-2020: Global Burden of Disease Study. Lancet 1997; 349: 1498-504.

5. Greenberg PE, Fournier AA, Sisitsky T. The economic burden of adults with major depressive disorder in the United States (2005 and 2010). J Clin Psychiatr 2015; 76(2): 155-162.

6. Katon $W$, Schulberg $H$. Epidemiology of depression in primary care. Gen Hosp Psychiatr 1992; 14: 237-47.

7. Simon GE, Korff M, Ludman EJ. Cost-effectiveness of a program to prevent depression relapse in primary care. Med Care 2002; 40: 941-950.

8. Simon GE. Evidence review: efficacy and effectiveness of antidepressant treatment in primary care. Gen Hosp Psychiatr 2002; 24: 213-24.

9. World Health Organization. Adherence to Long-Term Therapies: Evidence for Action. Geneva, Switzerland: World Health Organization, 2003.

10. Bobier C, Warwick M. Factors associated with readmission to adolescent psychiatric care. Aust $N Z \mathrm{~J}$ Psychiatr 2005; 39: 600-606.

11. Remien RH, Hirky $A E$, Johnson MO, Weinhardt $L S$, Whittier D, Le GM. Adherence to medication treatment: A qualitative study of facilitators and barriers among a diverse sample of HIV+ men and women in four US cities. AIDS Behav 2003; 7: 61-72.

12. Benson J, Britten N. Patients' decisions about whether or not to take antihypertensive drugs: qualitative study. $\mathrm{Br}$ Med J 2002; 325: 873-878.

13. Brody DS, Miller SM, Lerman CE, Smith DG, Caputo GC. Patient perception of involvement in medical care. J Gen Intern Med 1989; 4(6): 506-511.
14. Brown MT, Bussell JK. Medication adherence: WHO cares? Mayo Clinic Proceed Elsevier 2011; 86: 4-9.

15. Orlando M, Meredith LS. Understanding the causal relationship between patient-reported interpersonal and technical quality of care for depression. Med Care 2002; 40: 696-704

16. Alshammari $H$, Alaamer $K$, Albassam M, Aljumah $K$. The impact of decision aid on depressed patients' involvement in shared decision making: a Pilot randomized controlled double-blinded study. Pharmacotherapy. 111 River ST, Hoboken 07030-5774, NJ USA: Wiley-Blackwell; 2014

17. Elwyn $G$, Hutchings $H$, Edwards $A$, Rapport $F$, Wensing $M$, Cheung WY. The OPTION scale: measuring the extent that clinicians involve patients in decision-making tasks. Health Expect 2005; 8: 34-42.

18. Pampallona S, Bollini P, Tibaldi G. Patient adherence in the treatment of depression. Br J Psychiatr 2002; 180: 104-109.

19. Phatak A, Prusi R, Ward B. Impact of pharmacist involvement in the transitional care of high-risk patients through medication reconciliation, medication education, and postdischarge call-backs (IPITCH study). J Hosp Med 2016; 11: 39-44.

20. Klang SH, Ben-Amnon Y, Cohen Y. Community pharmacists' support improves antidepressant adherence in the community. Int Clin Psychopharmacol 2015; 30: 316-319.

21. Rubio-Valera M, Serrano-Blanco A, Magdalena-Belio J. Effectiveness of pharmacist care in the improvement of adherence to antidepressants: a systematic review and meta-analysis. Ann Pharmacother 2011; 45: 39-48.

22. Al-Jumah KA, Qureshi NA. Impact of pharmacist interventions on patients' adherence to antidepressants and patient-reported outcomes: a systematic review. BMC Psychiatr 2012; 6: 87-100.

23. Sawada N, Uchida $H$, Suzuki T. Persistence and compliance to antidepressant treatment in patients with depression: a chart review. BMC Psychiatr 2009; 16: 9: 38-43.

24. Bender KJ. Strategies to Improve Antidepressant Adherence. Addiction 2011; 7: 45-51.

25. Sansone RA, Sansone LA. Antidepressant Adherence. Are Patients Taking Their Medications? Innov Clin Neurosci 2012; 9: 41-46.

26. Tamburrino MB, Nagel RW, Chahal MK. Antidepressant Medication Adherence: A Study of Primary Care Patients. Prim. Care. Companion. J Clin Psychiatr 2009; 11: 205-211.

27. Alekhya $P$, Sriharsha $M$, Venkata $R$. Adherence to Antidepressant Therapy: Sociodemographic Factor Wise Distribution. Int J Pharm Clin Res 2015; 7: 180184.

28. Martín Vázquez MJ, García-Toro M, Campoamor F. Use and results of antidepressant treatment: patients' perception. Curr Drug Ther 2011; 6: 271-277. 
29. Martín Vazquez MJ, García-Toro M, Campoamor F. Uso de los tratamientos antidepresivos. La percepción del paciente. Actas Esp. Psiquiatr 2009; 37: 276-281.

30. Ching S, Chong HY, Chaiyakunapruk N. Clinical and economic impact of nonadherence to antidepressants in major depressive disorder: A systematic review. J Affect Disord 2016; 193: 1-10.

31. Keeley R, Smith M, Miller J. Somatoform symptoms and treatment nonadherence in depressed family medicine outpatients. Arch Fam Med 2000; 9: 46-54.

32. Zimmerman $M$, Posternak M, Friedman $M$. Which factors influence psychiatrists' selection of antidepressants? Am J Psychiatr 2004; 161: 1285-1289.

33. Sirey JA, Bruce ML, Alexopoulos GS. Perceived stigma as a predictor of treatment discontinuation in young and older outpatients with depression. Am J Psychiatr 2001; 158: 479-481.

34. Choi NG, Bruce ML, Sirrianni L. Self-reported antidepressant use among depressed, low-income homebound older adults: class, type, correlates, and perceived effectiveness. Brain Behav 2012; 2: 178-186.

35. Rossom RC, Shortreed S, Coleman KJ. Antidepressant adherence across diverse populations and healthcare settings. Depress. Anxiety 2016; 33: 765-774.

36. Oller-Canet S, Lacasta-Tintorer D, Castro Jl. Toman los pacientes deprimidos el tratamiento prescrito? Estudio descriptive sobre el cumplimiento del tratamiento antidepresivo. Actas. Esp. Psiquiatr 2011; 39: 288-293.

37. Jirón M, Escobar L, Arriagada L. Factores Asociados al Incumplimiento de los Tratamientos con Antidepresivos en Santiago, Chile. Value Health 2011; 14: 115-118.

38. Kane JM. Extending indications for long term pharmacotherapy: opportunities and challenges. Am J Psychiatr 2002; 159: 1-2.

39. Demyttenaere K, Haddad $P$. Compliance with antidepressant therapy and antidepressant discontinuation symptoms. Acta Psychiatr Scand Suppl 2000; 403: 50-56.

40. Stimmel G. Maximizing treatment outcome in depression: strategies to overcome social stigma and noncompliance. Dis Manag Health Outcome 2001; 9: 179186.

41. Bull SA, Hu XH, Hunkeler EM. Discontinuation of use and switching of antidepressants: influence of patient physician communication. J Am Med Assoc 2002; 288: 1403-1409.

42. Linden $M$, Gothe H, Dittmann RW. Early termination of antidepressant drug treatment. J Clin Psychopharm 2000; 20: 523-530.

43. Di Matteo MR. Enhancing patient adherence to medical recommendations. J Am Med Assoc 1994; 271: 79-84.

44. Párraga I, López-Torres J, del Campo JM. Seguimiento de la adherencia al tratamiento antidepresivo en pacientes que inician su consumo. Aten Primaria 2014; 46: 357-366.

45. Unützer J, Katon W, Callahan CM. Depression treatment in a sample of 1801 depressed older adults in primary care. J Am Geriatr Soc 2003; 51: 505-514.
46. Krivoy $A$, Balicer RD, Feldman B. Adherence to Antidepressants is associated with lower mortality: a 4year population-based cohort study. J. Clin. Psychiatr 2016; 77: e566-e572.

47. Di Matteo MR, Lepper HS, Croghan TW. Depression is a risk factor for noncompliance with medical treatment: meta-analysis of the effects of anxiety and depression on patient adherence. Arch Intern Med 2000; 160: 2101 2107.

48. McDonald HP, Garg AX, Haynes RB. Interventions to enhance patient adherence to medication prescription: Scientific review. J Am Med Assoc 2002; 288: 28682879.

49. Pampallona S, Bollini P, Tibaldi G. Patient adherence in the treatment of depression. Br J Psychiatr 2002; 180: 104-109.

50. Chong WW, Aslani $P$, Chen TF. Effectiveness of interventions to improve antidepressant medication adherence: a systematic review. Int J Clin Pract 2011; 65: 954-975

51. Aljumah K, Hassali MA. Impact of pharmacist intervention on adherence and measurable patient outcomes among depressed patients: a randomized controlled study. BMC Psychiatr 2015; 15: 8-13.

52. Finley PR, Rens HR, Pont JT. Impact of a collaborative pharmacy practice model on the treatment of depression in primary care. Am J Health Syst Pharm 2002; 59: 1518.

53. Al-Saffar N, Deshmukh AA, Carter P. Effect of information leaflets and counselling on antidepressant adherence: open randomised controlled trial in a psychiatric hospital in Kuwait. Int J Pharm Pract 2005; 13: 123-131.

54. Rubio-Valera M, March Pujol M, Fernandez A. Evaluation of a pharmacist intervention on patients initiating pharmacological treatment for depression: a randomized controlled superiority trial. Eur Neuropsychopharmacol 2013; 23(9): 1057-1066.

55. Capoccia KL, Boudreau DM, Blough DK. Randomized trial of pharmacist interventions to improve depression care and outcomes in primary care. Am J Health Syst Pharm 2004; 61: 364-372.

56. Adler DA, Bungay KM, Wilson IB. The impact of a pharmacist intervention on 6-month outcomes in depressed primary care patients. J Health 2004; 26: 199-209.

57. Finley PR, Rens HR, Pont JT. Impact of a collaborative care model on depression in a primary care setting: a randomized controlled trial. Pharmacother $J$ Hum Pharmacol Drug Ther 2003; 23: 1175-1185.

58. Rickles NM, Svarstad BL, Statz-Paynter JL. Pharmacist telemonitoring of antidepressant use: effects on pharmacist-patient collaboration. J Am Pharm Assoc JAPhA 2005; 45: 344-353.

59. Crockett J, Taylor S, Grabham A. Patient outcomes following an intervention involving community pharmacists in the management of depression. Aust $J$ Rural Health 2006; 14: 263-269.

Trop J Pharm Res, February 2019; 18(2): 442 
60. Brook $\mathrm{OH}$, Hout $H$, Stalman W. A pharmacy-based coaching program to improve adherence to antidepressant treatment among primary care patients. Psychiatr Serv 2005; 56: 487-489.

61. Marques LA, Galduroz JC, Fernandes MR. Assessment of the effectiveness of pharmacotherapy follow-up in patients treated for depression. J Manag Care Pharm 2013; 19(3): 218-227.

62. Ho SC, Chong HY, Chaiyakunapruk N. Clinical and economic impact of non-adherence to antidepressants in major depressive disorder: a systematic review. $J$ Affect Disord 2016; 193: 1-10.

63. Bower P, Gilbody S, Richards D. Collaborative care for depression in primary care: making sense of a complex intervention: systematic review and meta-regression. $\mathrm{Br}$ J Psychiatr 2006; 189(6): 484-493.

64. Smith M, Bates DW, Bodenheimer TS. Pharmacists belong in accountable care organizations and integrated care teams. Health Aff 2013; 32: 1963-1970. 